\title{
Estimation of microwave resonant measurement uncertainty from uncalibrated data
}

\author{
K. Torokhtii ${ }^{1}$, A. Alimenti ${ }^{1}$, N. Pompeo ${ }^{1}$, E. Silva ${ }^{1}$ \\ ${ }^{1}$ Department of Engineering, Roma Tre University, Via Vito Volterra, 62, 00146 Roma, Italy
}

ABSTRACT

We present an extended study on the uncertainty in resonant measurements. The uncertainty of the resonant frequency and quality factor has been estimated. Two different measurement systems and different fitting approaches are used. The effect of the use of uncalibrated resonant curves on uncertainty has been extensively studied. For the uncalibrated data, the systematic contribution to the uncertainty is determined.

\section{Section: RESEARCH PAPER}

Keywords: dielectric resonator; microwaves; uncertainty

Citation: Kostiantyn Torokhtii, Andrea Alimenti, Nicola Pompeo, Enrico Silva, Estimation of microwave resonant measurements uncertainty from uncalibrated data, Acta IMEKO, vol. 9, no. 3, article 8, September 2020, identifier: IMEKO-ACTA-09 (2020)-03-08

Editor: Jan Saliga, Technical University of Košice, Slovakia

Received January 17, 2020; In final form April 21, 2020; Published September 2020

Copyright: This is an open-access article distributed under the terms of the Creative Commons Attribution 3.0 License, which permits unrestricted use, distribution, and reproduction in any medium, provided the original author and source are credited.

Corresponding author K. Torokhtii, e-mail: kostiantyn.torokhtii@uniroma3.it

\section{INTRODUCTION}

Continuous interest in microwave measurements has led to the realisation of industrial grade methods for material characterisation. A wide range of material properties could be characterised at microwaves. One of the advantages of microwave techniques is their high sensitivity. They are widely used for the measurement of the complex permittivity of liquids [1] and solid dielectrics [2]. Traditionally, microwave techniques have been used for the surface impedance (of $Z_{S}$ ) measurements of conductors. In particular, the microwave Dielectric Resonator (DR) method is a standard for the measurements of $Z_{S}$ of superconductors [3]. In connection with other techniques as DC measurements, the DR method can show a detailed picture of the microscopic properties of superconductors [4], [5]. In this article, we focus on the DR-based measurement technique.

In recent years, Vector Network Analysers (VNAs) have become more accessible due to their low cost [6], [7]. However, the quality of the measurements relies heavily on the calibration procedure. In the case of low-end VNAs, the importance of calibration becomes critical.

In some cases, calibration becomes an impossible operation. One notable case is when the Device Under Test (DUT) is placed in a harsh or particularly difficult environment. A representative example is given by measurements of materials or devices at low, cryogenic temperatures [8], [9]. Apart from the complexity of the cryogenic measurement system, one of the main problems originates from the part of the microwave line inside the cryostat, which cannot be calibrated using the standard procedure. Here, the main role is taken by the absence of microwave standards that are capable of maintaining their characteristics in a hostile environment. One possible solution is to develop custom standards [10], but this often requires very advanced techniques and specific expertise. Moreover, it is rather difficult to associate the appropriate uncertainty with the measured quantity.

In this article, we extend the analysis of the effect of the uncalibrated measurements [11], [12] to a more complete generalised picture of the resonant parameters. We focus our attention on the resonant frequency $\left(f_{0}\right)$ and quality factor $(Q)$. Here, we explore the impact of different VNAs and fitting methods on the estimation of uncertainties on $Q$ and $f_{0}$. A key objective of the study is the determination of the uncertainty of $Q$ and $f_{0}$ for measurements where no calibration could be performed. For the measurements, we use the Hakki-Coleman $(\mathrm{H}-\mathrm{C})$ dielectric resonant system described in [11], [12].

\section{VARIABLE-Q RESONATOR CELL}

In this study, we aim to evaluate the measurement uncertainty on $Q$ and $f_{0}$ when a microwave line, or a part thereof, cannot be calibrated, as in the cryogenic scenario outlined above. We designed an 'open' type H-C resonator, operating at room temperature. In our experiment, we used phase stable microwave cables with female-female connectors. We designed the DR cell 
to have the reference plane of the microwave cable connector near the edge of the cell. Coupling was made by removable antennas inserted directly in the cable connector. With this design, we were able to perform the calibration up to the reference plane of the cable connectors. The DUT is therefore the resonator cell only, as is desirable in an ideal setup. In this way, we are able to directly compare the uncalibrated measurements with the calibrated ones - without any unaccounted-for contribution and without the need for a deembedding procedure [13].

In Figure 1, a sketch of the measurement cell is shown. The dielectric resonator is cylindrical and loaded with a sapphire puck, chosen because of its low dielectric losses and high permittivity. The latter allows us to concentrate the electromagnetic field within and near the dielectric puck. A mono-crystal sapphire puck with a diameter of $(8.0 \pm 0.1) \mathrm{mm}$ and a height of $(4.5 \pm 0.1) \mathrm{mm}$ was used. An Anritsu 37269D VNA and a R\&S ZVA-40 VNAs with phase-stable Anritsu cables were used for measurements and connections.

We are interested in an evaluation of the uncertainty in the measurements of the resonator parameters with different quality factors $Q$. We then designed a system for measurements in a wide range of $Q$ factors in the same conditions, which include, in particular, the frequency range of operation of the DR.

In order to change the $Q$ values of the resonator in a controlled way, we could substitute the bases using metals with different resistivities and change the position of the upper base, thereby changing the height of the corresponding gap of the DR. By fine tuning the latter, we were able to keep the resonant frequency within a chosen fixed range, $(13.0 \pm 0.5) \mathrm{GHz}$.

\section{Q-FACTOR AND RESONANT FREQUENCY EXTRACTION}

$Q$ and $f_{0}$ are important properties of a resonator. A good example is the resonant technique to measure the surface impedance of a material $Z_{\mathrm{S}}=R_{\mathrm{S}}+i X_{\mathrm{S}}$, where $R_{\mathrm{S}}$ and $X_{\mathrm{S}}$ are the surface resistance and surface reactance, respectively. By using this measurement technique, the quality of the measurements of the surface impedance by the resonant method depends directly on $f_{0}$ and $Q$. Usually, the variation of the surface impedance with some external parameter is obtained experimentally, while the determination of the absolute value of $Z_{\mathrm{S}}$ is a more complex issue. The relationship between $Z_{\mathrm{S}}$ and resonant parameters is the following [14]:

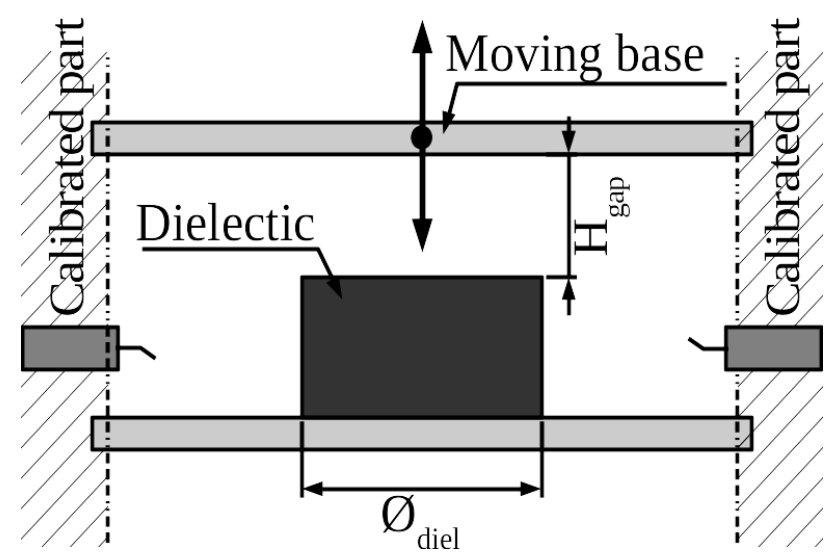

Figure 1. Variable- $Q$ resonant cell.
$\Delta Z_{\mathrm{S}}=\Delta R_{\mathrm{S}}+i \Delta X_{\mathrm{S}}=G \Delta \frac{1}{Q}-2 i G \frac{\Delta f_{0}}{f_{0}}+b g$,

where $G$ is a constant called geometrical factor; the background ' $b g$ ' is the contribution of the resonator without the sample; and $\Delta x$ is the variation of the parameter $x$ with respect to the reference value. From Equation (1), it can be seen that the uncertainty of $Z_{\mathrm{S}}$ depends on $u(Q), u\left(f_{0}\right)$, and $(u(G)$. The latter, usually $<1 \%$, can be obtained through electromagnetic simulations. The remaining uncertainties, $u(Q)$ and $u\left(f_{0}\right)$, are not readily available when no calibration is possible: hence the interest in the estimate of $u(Q)$ and $u\left(f_{0}\right)$ is evident.

In a resonator working in transmission, $f_{0}$ and $Q$ can be obtained from the measurements of the response of a two-port microwave resonant system, described through a $2 \times 2$ scattering matrix [S] [15]. In particular, the most reliable determination of the resonant parameters could be obtained from the complexvalued transmission coefficient, $S_{\operatorname{tr}}\left(S_{12}\right.$ or $\left.S_{21}\right)$. While preliminary estimates for $Q$ and $f_{0}$ can be obtained by the socalled $-3 \mathrm{~dB}$ method, a more precise approach is to use an appropriate fit of the resonance curve. In [16], it was shown that a Lorentzian curve can be used to fit the function $\left|S_{\mathrm{tr}}(f)\right|^{2}$ only in 'ideal' cases. The real resonator includes corrections connected to cross-coupling and phase contributions. The resonance curve can be described through the following equation (the Fano resonance model [17]) with a phase correction:

$S_{\mathrm{tr}}(f)=\left[\frac{S_{\mathrm{tr}}\left(f_{0}\right)}{1-2 i Q \frac{f-f_{0}}{f_{0}}}+S_{\mathrm{c}}\right] e^{i \varphi}$,

where $S_{\mathrm{tr}}\left(f_{0}\right)>0$ is the transmission $S$-parameter at the resonant frequency, and $S_{\mathrm{c}}$ represents the contribution originated from the cross-coupling between the resonator ports and $e^{i \varphi}$, with $\varphi=\alpha+\beta f$, taking into account the propagation phase delay along the line.

There is a large number of methods for the extraction of $f_{0}$ and $Q$ from the experimental data [18]. A widely used method is the so-called circular fit approach [19], but it is also possible to use a complex-valued modification of the Levenberg-Marquardt algorithm to fit the complex $S_{\text {tr }}$ [20]. However, complex-valued fitting requires more calculation power and custom algorithms. Additional complexities arise when the uncertainty of the fitting parameters must be estimated. From this point of view, the fit of the modulus of $S_{\mathrm{tr}}$ remains more accessible due to already implemented algorithms in popular programming languages such as Python and MATLAB [21]. Using the fit of $\left|S_{\mathrm{tr}}(f)\right|$, one can implement a fitting procedure with cheap computers like the Raspberry Pi. $\left|S_{\mathrm{tr}}(f)\right|$ is obtained from Equation (2) as:

$$
\begin{aligned}
\left|S_{\mathrm{tr}}(f)\right|^{2}=\frac{S_{\mathrm{tr}}\left(f_{0}\right)}{1+} & 4 Q^{2}\left(\frac{f-f_{0}}{f_{0}}\right)^{2} \\
+ & 2 \operatorname{Re}\left(S_{\mathrm{c}}\right) \\
+ & 4 Q \frac{f-f_{0}}{f_{0}} \operatorname{Im}\left(f_{0}\right) \\
+ & \operatorname{Re}^{2}\left(S_{\mathrm{c}}\right)+\operatorname{Im}^{2}\left(S_{\mathrm{c}}\right) .
\end{aligned}
$$

This fit requires only five independent parameters.

Since a VNA allows the measurement of the $S$-parameters as complex quantities, a fit of the complex quantity $S_{\mathrm{tr}}(f)$ could 
also be devised, yielding also the additional information concerning the phase correction $e^{i \varphi}$. Hence, differently to [12], we additionally perform a complex fit of Equation (2) and compare the results that can be obtained using both approaches. Resorting to standard algorithms, which usually accept only real quantities in input, we use a specific approach to fit complex data and include experimental data uncertainty $\left(u\left(S_{\text {tr }}\right)\right)$. In order to do so, we define the objective function to be minimised by separating the real and imaginary parts of $S_{\mathrm{tr}}$ - each with its experimental uncertainty. Hence, each measured $S_{\text {tr }}$ curve, composed of $n$ data points, becomes an array of $2 n$ data points - with the first half given by the real parts and the second by the imaginary parts. Correspondingly, the fit function is given by the real and imaginary parts of Equation (2) for the first and second half of the measurement data set, respectively. Consequently, differently to the fit to Equation (3), the fit is done with seven independent parameters.

In summary, the uncertainties $u(Q)$ and $u\left(f_{0}\right)$ become functions of various parameters and conditions, such as the $S$ parameters' uncertainty, contributions from the fit algorithm $\left(\mathbb{C}_{\text {fit }}\right)$, and the presence of the calibration $\left(\mathbb{C}_{\text {cal }}\right) u(Q)=$ $\mathcal{F}_{Q}\left(u\left(S_{\text {tr }}\right), \mathbb{C}_{\text {fit }}, \mathbb{C}_{\text {cal }}\right)$ and $u\left(f_{0}\right)=\mathcal{F}_{f 0}\left(u\left(S_{\text {tr }}\right), \mathbb{C}_{\text {fit }}, \mathbb{C}_{\text {cal }}\right)$.

\section{MEASUREMENT PROCEDURE}

In this section, we present an extended elaboration of the measurement procedure presented in [11]. The aim is to complete the estimate of the uncertainty of the measurements of the characteristic parameters of a resonator for uncalibrated measurements, including the resonant frequency.

We performed experimental measurements of the transmission coefficient $S_{\mathrm{tr}}(f)$ corresponding to a wide range of $Q$ factors, namely 1500 - 9500, with very small variations of other controlled parameters of the resonant system. The interest is the comparison between the $Q$ and $f_{0}$ measurements obtained from the fit of $S_{\mathrm{tr}}(f)$ with and without the VNA calibration. Measurements were done using two different VNAs (the Anritsu 37269D and the R\&S ZVA-40) to assess the robustness of the results among different instruments. Indeed, this point is not trivial, since the uncalibrated curves contain contributions that also originate from the internal VNA circuitry. In calibrated measurements, the VNA circuitry nonidealities are transparently removed.

Firstly, we fixed the calibration in the narrow frequency band 12.5-13.5 GHz, which was chosen to accommodate all the resonant curves (with different $Q$ ) requiring measurement with sufficient density of frequency points. Measurements were performed using frequency sweeps with 1601 calibrated points with the Anritsu 37269D VNA (the maximum number of data points) and 32000 calibrated points with the R\&S ZVA-40. The calibration was performed by the standard 12-term Short-OpenLoad-Thru (SOLT) procedure by the algorithms incorporated in both VNAs. For the experimental measurements, we followed the Anritsu 37269D and R\&S ZVA-40 standard calibration guides. The series of resonance curves were then recorded, varying the $Q$-factor in the range $1500<Q<9500$. In order to obtain such a wide range of $Q$ with the same setup, we changed the air gap and we used different metals for the bases, as we explained before. The gap, controlled by only one movable part of the resonator cell - the upper base (Figure 1) - allowed us to tune the operating resonance frequency to be always within the frequency range of the calibration.

For each fixed position of the upper base, two measurements were performed: one with and one without the calibration. In Figure 2, an example of the measured calibrated and uncalibrated resonant curves is shown for Anritsu 37269D (left panels of Figure 2) and R\&S ZVA-40 (right panels of Figure 2). The difference is immediately appreciated, confirming the need to assess the quality of an uncalibrated measurement even for a resonant device (naïvely, one could expect that resonant systems are sufficiently narrowband such that they are almost insensitive to the calibration).

\section{RESULTS}

A series of $S_{\mathrm{tr}}(f)$ resonant curves $(\sim 40)$ was recorded and fits by Equation (1) (complex fit) and Equation (2) (fit of the modulus) were applied to the uncalibrated and calibrated data. Care was taken to maintain similar fit conditions. It should be noted that in our situation, in each curve, the measured points were taken at evenly spaced frequencies for all measurements. Since $Q$ changes significantly, without proper 'normalizations', the fits of the curves with low and high $Q$ would not be in the same conditions concerning the density of information per interval.

Therefore, we proceeded as follows. Firstly, we limited the span of each curve to $N \Delta f_{\mathrm{FWHM}}$, where $\Delta f_{\mathrm{FWHM}}$ is the full-width half-maximum frequency range, and $N>1$ is a proper multiplicative factor. In our case, $N$ was constrained by the available frequency range of the calibration and the requirement to be the same for all experimental curves. As a result, we chose $N=4$. The resulting curves, which have different frequency spans with evenly spaced frequency points, had different numbers of points in this step. Each set was then trimmed uniformly in order to maintain the same amount of evenly spaced data points as much as possible. As a result, we obtained the final set of the resonant curves with $Q$ in the range of $1500-9500$, trimmed and with same information density, with the span $4 \Delta f_{\mathrm{FWHM}}$, and with the number of data points near 300 . We now discuss the result of the elaboration of this set of curves.
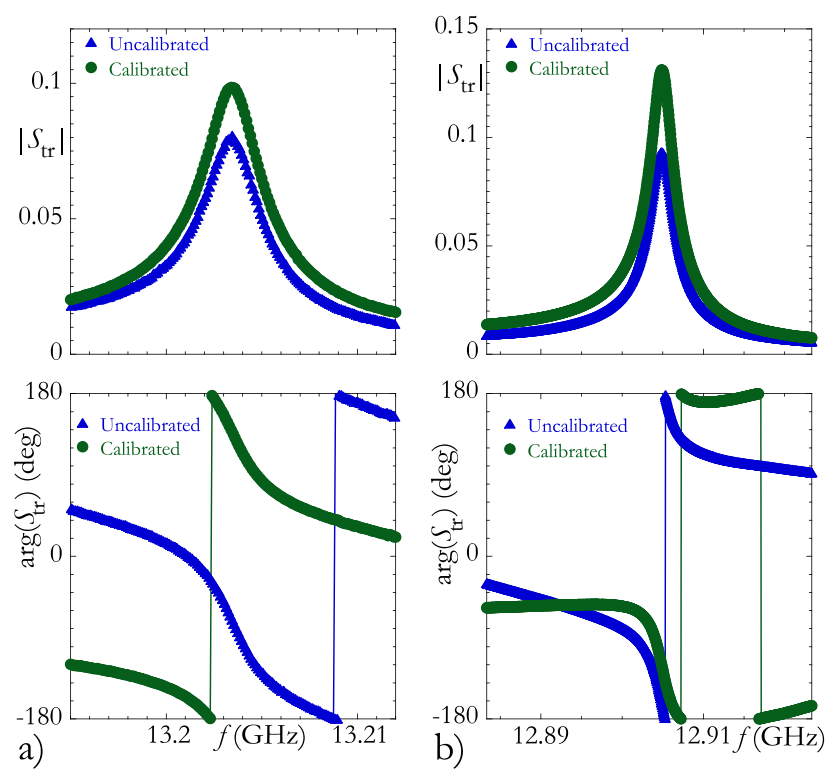

Figure 2. The measured resonant curves with and without calibration applied in the modulus (upper panels) and phase (lower panels). Panel (a) Anritsu VNA. Panel (b) R\&S VNA. 
Concerning the uncertainties, it should be noted that the uncertainty $u\left(S_{\mathrm{tr}}\right)$ could only be estimated for the calibrated curve [12]. In particular, for the Anritsu 37269D, these estimations could be given by the Exact Uncertainty Calculator - a proprietary software tool of Anritsu [22] (although alternative tools like in [23] could be used). This tool automatically estimates the uncertainties for all $S$-parameters, taking into account the characteristics of the VNA, cables, connectors, and calibration kit. For R\&S ZVA-40, on the other hand, the uncertainty was obtained from the general specifications of the VNA provided in the datasheets. Hence, the uncertainty for R\&S ZVA-40 is not accurately tailored to the measurements and should be considered as an over-estimated, worst-case figure. For both VNAs, the estimation of $u\left(S_{\mathrm{tr}}\right)$ is provided in the form of dependencies of the uncertainties of $u\left(\left|S_{\mathrm{tr}}\right|\right)$ and $u\left(\arg \left(S_{\mathrm{tr}}\right)\right)$ on $\left|S_{\mathrm{tr}}\right|$. Thus, separate uncertainties should be assigned for each measured point, yielding additional complications in the fit procedure. Moreover, since the uncertainties are provided on the modulus and phase, the uncertainties on the real and imaginary parts of $S_{\text {tr }}$ needed for the complex fit were derived by the uncertainty propagation. In the calibrated conditions, the worst case of the measurement uncertainty on $S_{\mathrm{tr}}$ was $<1.7 \%$ for the modulus and $<0.5 \%$ for the phase for Anritsu 37269D. The worst-case measurement uncertainties for R\&S ZVA-40 were $<2.4 \%$ for the modulus and $<1.6 \%$ for the phase. For the uncalibrated curves, zero uncertainty on $S_{\text {tr }}$ had to be used in the absence of the necessary information.

We limited our study scope to already implemented solutions only. A widely used Levenberg-Marquardt [20] fit algorithm implementation in the Python ScyPy package (curvefit) applied a MINPACK code to perform a reliable fit. The fit procedure used here provides a covariance matrix based on the numerically computed Jacobian $(J)$ - based on the modified model function. The covariance matrix is $\operatorname{Cov}=\left(R^{T} R\right)^{-1}$, where $R=r P, r$ is the upper triangle of $J$, and $P$ is a permutation matrix (more
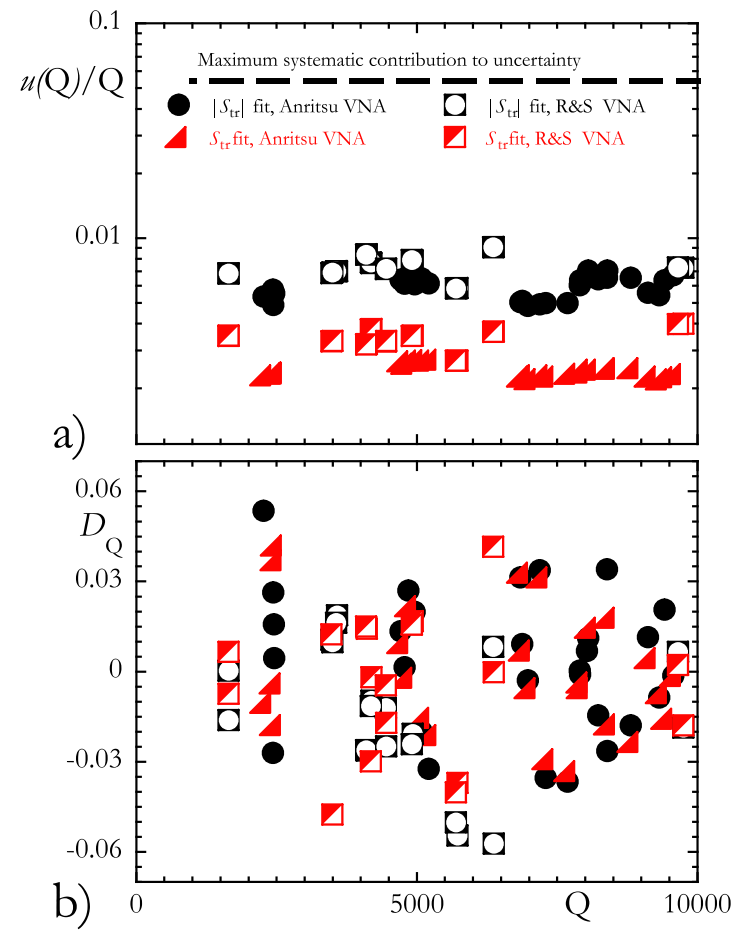

Figure 3. Panel (a) Relative uncertainty on calibrated $Q$-factor vs $Q$. Panel (b) Discrepancy $D_{Q}$ vs $Q$. details regarding fit algorithm can be found in [21], [24]). The square root of the diagonal elements of the $\mathrm{Cov}$ matrix yields an estimate of the uncertainties of the fit parameters. In this way, the uncertainties of $Q$ and $f_{0}$, as fit parameters can be estimated.

Moreover, as a consequence of the non-application of the calibration, additional significant sources of uncertainty are expected. Thus, we define as a measure of this uncertainty (for the uncalibrated measurements) the discrepancy between the resonant parameters as derived by the fitting of the calibrated and uncalibrated curves. The discrepancy of $Q$ can be defined as the relative variation between $Q$ obtained from calibrated $\left(Q_{\text {cal }}\right)$ and uncalibrated $\left(Q_{\text {uncal }}\right)$ data, $D_{Q}=\left(Q_{\text {uncal }}-Q_{\text {cal }}\right) / Q_{\text {cal }}$. For frequency $f_{0}$, the discrepancy is defined in the same manner as

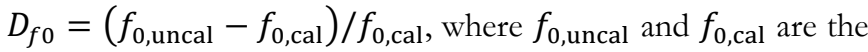
resonant frequencies obtained by the fit of the uncalibrated and calibrated curves, respectively.

In Figure 3 and Figure 4, we show the main results of the study with the aim of comparing the two fitting approaches. In order to fully exploit the available data, both the scattering coefficients $S_{21}$ and $S_{12}$, simultaneously measured for each resonator configuration, are fitted as $S_{\mathrm{tr}}$. Indeed, although the resonator itself is a reciprocal device, the same does not hold true for the whole line due to the inevitable asymmetry of the lines between the resonator and VNAs, so $S_{21}$ and $S_{12}$ are different.

The uncertainties of the uncalibrated measurements (because in this case, $u\left(S_{\mathrm{tr}}\right)=0$ was taken) include only the contribution of the uncertainty arising from the curve fit process, as provided by the fit algorithms. This leads to a relatively low uncertainty for $Q$ and $f_{0}$. Very approximately, based on the available number of measured curves, an estimation of the relative uncertainty of the experimental uncalibrated data gives median values for $u\left(f_{0}\right) / f_{0}$ between $0.05 \mathrm{ppm}$ and $0.18 \mathrm{ppm}$ and for $u(Q) / Q$ between $0.08 \%$ and $0.17 \%$ (see Table 1 ). Comparing the results with the two different fit approaches, i.e. of the complex $S_{\mathrm{tr}}$ and of its modulus, we obtain similar levels of the uncertainties as provided

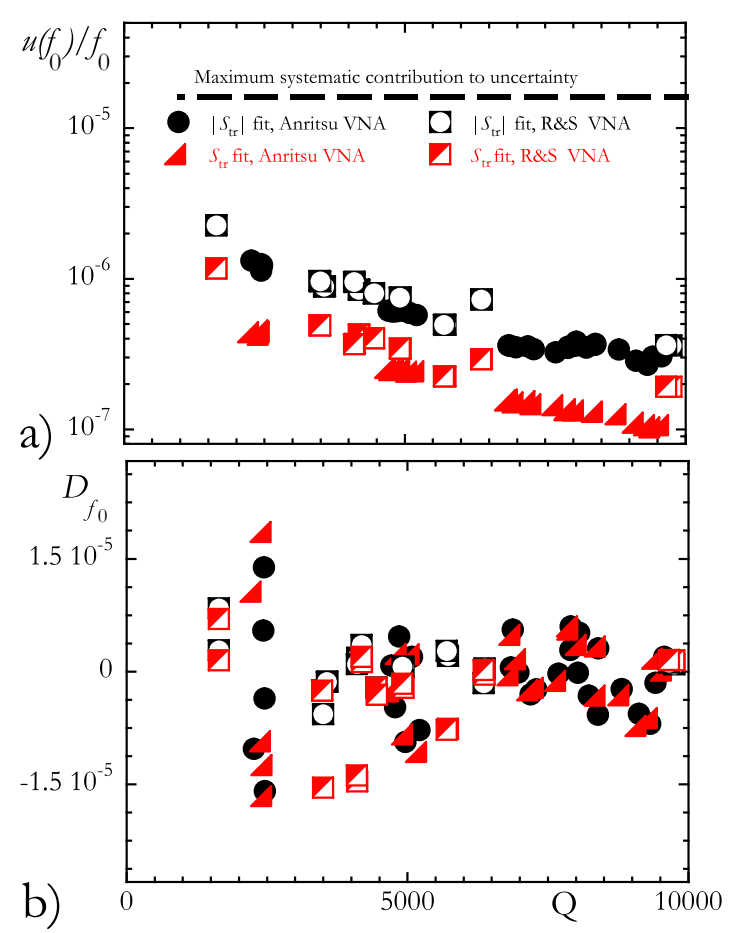

Figure 4. Panel (a) Relative uncertainty on calibrated $f_{0}$ vs $Q$. Panel (b) Discrepancy $D_{f 0}$ vs $Q$. 
Table 1. Median values of the relative uncertainties of $Q$ and $f_{0}$ of experimental uncalibrated data.

\begin{tabular}{cccc}
\hline & Anritsu 37269D VNA measurement & R\&S ZVA-40 VNA measurement & $\boldsymbol{u}(\boldsymbol{Q}) / \boldsymbol{Q}\left(\boldsymbol{f}_{\mathbf{0}}\right) / \boldsymbol{f}_{\mathbf{0}}$ \\
\hline Fit by Equation $(3)\left(\left|S_{\mathrm{tr}}\right|\right)$ & $\boldsymbol{u}\left(\boldsymbol{f}_{\mathbf{0}}\right) / \boldsymbol{f}_{\mathbf{0}}$ & 0.132 & 0.140 \\
Fit by Equation $(2)\left(S_{\mathrm{tr}}\right)$ & 0.074 & 0.083 & 0.141 \\
\hline
\end{tabular}

by the fit algorithms. Moreover, since these are well below the error produced by the absence of the calibration, when dealing with uncalibrated measurements, the simpler fit of the modulus of $S_{\mathrm{tr}}$ is certainly adequate and avoids unnecessary numerical complications and longer fit times.

Considering the calibrated data, the uncertainties of $Q$ and $f_{0}$ become more than two times higher than in the uncalibrated case because of the additional contribution of the known $u\left(S_{\mathrm{tr}}\right) \neq 0$. In the following discussion, we focus our attention on the calibrated data. In Figure 3(a) and Figure 4(a), the relative uncertainties $\left(u_{r}\right)$ of the $Q$-factors and resonant frequencies $f_{0}$ of the calibrated data are shown.

Comparing the results of the two fitting approaches, we observe that apart from providing the additional important information about the phase correction, the fit of the complex $S_{\text {tr }}$ data is in overall characterised by a $\sim 1.5$ times lower $u_{r}$ on the fitting parameters. From Figure $3(\mathrm{a})$, it is clear that the maximum $Q$-factor uncertainty reduces from $u(Q) / Q<0.7 \%$ for the fit of $\left|S_{\text {tr }}\right|$ to $u(Q) / Q<0.3 \%$ for the fit of the complex $S_{\mathrm{tr}}$. Additionally, it can be seen that $u_{r}(Q)$ is almost independent of the $Q$-factor value. Moreover, reasonably, $u(Q) / Q$ is also independent of the VNA used for the measurement. Concerning the relative uncertainty of $f_{0}$, the fit of $\left|S_{\text {tr }}\right|$ yields $u\left(f_{0}\right) / f_{0}<1.5 \mathrm{ppm}$ (Figure $4(\mathrm{a})$ ), which becomes lower than $0.5 \mathrm{ppm}$ using the fit of the complex $S_{\mathrm{tr}}$.

The comparison of the fit parameters between the calibrated and uncalibrated cases can be explained using the discrepancy parameter $D$, reported in Figure 3(b) and Figure 4(b) for both VNAs used in measurement and for the two fitting approaches (black markers for $\left|S_{\mathrm{tr}}\right|$ and red markers for fit of $S_{\mathrm{tr}}$ ). Firstly, since the absolute values of the discrepancy are, on average, larger than the uncertainties of the calibrated measurements, we can infer that they are indeed a good measure of the error due to the use of uncalibrated data.

Moreover, within the relatively large set of experimental curves ( 40), it can be observed (Figure 3(b) and Figure 4(b) use the same legend as in the 'a' subfigures) that the discrepancy values have a balanced distribution around zero for both fit approaches. This feature calls for a closer investigation of the characteristic of this distribution, allowing us to go beyond a rough estimation of the error due to the use of uncalibrated curves as worst-case values only [10]. The latter yields, as an estimate of the maximum additional contribution to the measurement relative uncertainty, arising from the use of uncalibrated data (equal to $5.4 \%$ for $Q$ and to $16 \mathrm{ppm}$ for $f_{0}$ ), but we show below that the data supports a reduction of this figure.

We thus gain further information by making use of the finding, as depicted in Figure 3(b) and Figure 4(b), that the discrepancy is independent of the measuring device (which is not obvious, since we are dealing with uncalibrated data). In the following section, we combine all the data from Figure 3(b) and Figure 4(b) for the observed discrepancies for data obtained with Anritsu 37269D and R\&S ZVA-40 in order to compare the fit approaches.
Analysing the statistical distribution of the $D$ values, reported in the histograms in Figure 5(a) and Figure 5(b) for $Q$ and $f_{0}$ respectively, it can be seen that they yield relatively symmetric shapes centred almost at zero. In particular, for the discrepancy in $f_{0}$, its absolute mean value is $0.44 \mathrm{ppm}$, which is due to the fit of $\left|S_{\mathrm{tr}}\right|$ (black histogram) and $1.9 \mathrm{ppm}$ for the $S_{\mathrm{tr}}$ fit (red histogram), close to the upper value of the corresponding uncertainty of $u\left(f_{0}\right) / f_{0}$ in the calibrated data. The absolute mean values of the quality factor discrepancies are less than the uncertainty of $u(Q) / Q$ for the calibrated data: $0.38 \%$ for the fit of $\left|S_{\mathrm{tr}}\right|$ and $0.16 \%$ for the $S_{\mathrm{tr}}$ fit.

Interestingly, for the two fitting approaches, the standard deviations of $D$ are similar: $\sim 6 \mathrm{ppm}$ and $\sim 2.2 \%$ for the discrepancies in $f_{0}$ and $Q$, respectively. It can be noted that the discrepancy distributions have a dispersion (as measured by the standard deviation) that is much larger than their centre values (given by the mean values). This allows us to obtain a refined estimation of the overall uncertainty by resorting to the standard deviations of the discrepancy distributions with respect to the above-mentioned worst-case values.

\section{CONCLUSIONS}

We performed an extended study of the measurement uncertainty on the quality factor and resonant frequency $f_{0}$ of
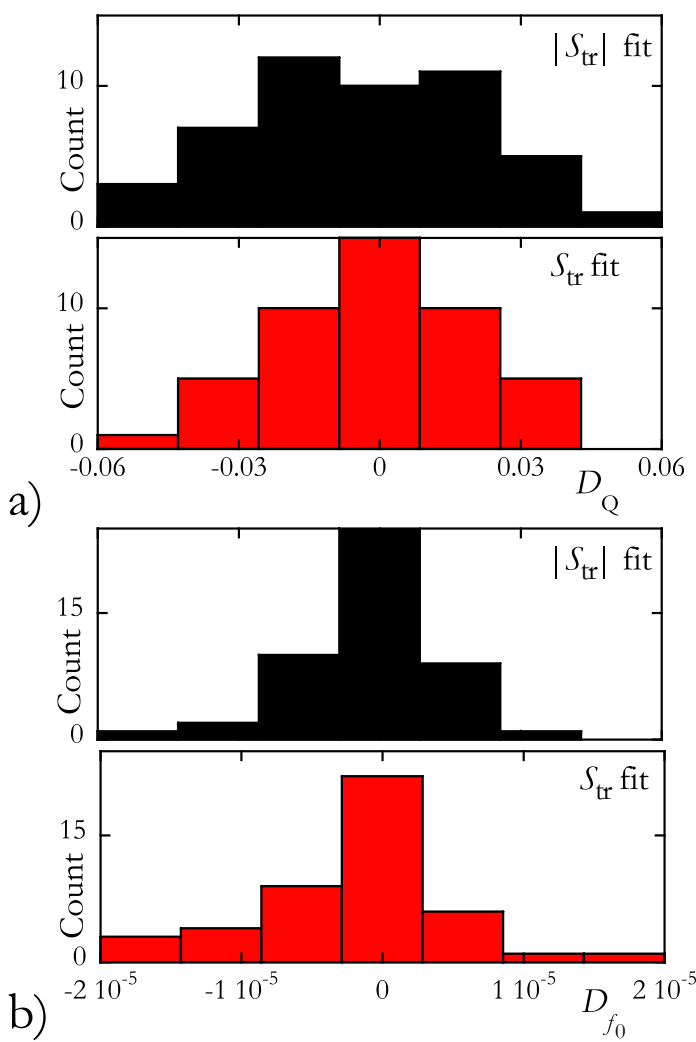

Figure 5. Distribution of the discrepancy $D_{\mathrm{Q}}$ (panel a) and $D_{f 0}$ (panel b). 
the microwave resonator, which arises when the microwave line is not calibrated. By exploring a wide range of $Q$, enabled by the implementation of an ad-hoc resonator cell, we compared the obtained results with calibrated and uncalibrated data.

We used different VNAs for data acquisition following the same procedure and different fit methods. After proper 'normalisations' of the datasets, in terms of frequency span and point number, we find that while the worst-case uncertainty in the calibrated curves is below $0.75 \%$ for $Q$ and below $1.5 \mathrm{ppm}$ for $f_{0}$, in the uncalibrated measurements, an additional contribution arises.

By estimating this contribution from the discrepancy between the fit results of the calibrated and uncalibrated data, we obtain an experimental quantification of the additional contribution that is equal to $5.4 \%$ for $Q$ and $16 \mathrm{ppm}$ for $f_{0}$ as a maximum error. By combining all the data for the discrepancy, we can reduce these figures to $2.2 \%$ and $6 \mathrm{ppm}$, respectively, which now should be intended as the standard uncertainties. These results provide a guide for the evaluation of the uncertainty contribution, which should be taken into account when the calibration of microwave lines similar to the one studied is not feasible.

\section{REFERENCES}

[1] S. Chen, M. Guo, K. Xu, P. Zhao, Y. Hu, L. Dong, G. Wang, A dielectric constant measurement system for liquid based on SIW resonator, IEEE Access 6 (2018) pp. 41163-41172. Online [accessed 25 September 2020] DOI: https://doi.org/10.1109/ACCESS.2018.2857514

[2] J. W. Schultz, A new dielectric analyzer for rapid measurement of microwave substrates up to $6 \mathrm{GHz}$, Proc. of AMTA 2018, Williamsburg, 4-9 November 2018, pp. 1-6.

[3] IEC 61788-7:2006, Superconductivity - Part 7: Electronic characteristic measurements - Surface resistance of superconductors at microwave frequencies.

[4] E. Bartolomé, F. Vallés, A. Palau, V. Rouco, N. Pompeo, F. F. Balakirev， B. Maiorov， L. Civale，T. Puig， X. Obradors, E. Silva, Intrinsic anisotropy versus effective pinning anisotropy in $\mathrm{YBa} 2 \mathrm{Cu} 3 \mathrm{O} 7$ thin films and nanocomposites, Phys. Rev. B 100 (2019) Art. ID. 054502.

DOI: https://doi.org/10.1103/PhysRevB.100.054502

[5] N. Pompeo, A. Augieri, K. Torokhtii, V. Galluzzi, G. Celentano, E. Silva, Anisotropy and directional pinning in $\mathrm{YBa}_{2} \mathrm{Cu}_{3} \mathrm{O}_{7-\mathrm{x}}$ with $\mathrm{BaZrO}_{3}$ nanorods, Appl. Phys. Lett. 103 (2013) Art. ID. 022603. DOI: https://doi.org/10.1063/1.4813405

[6] K. Will, T. Meyer, A. Omar., Low-cost high-resolution handheld VNA using RF interferometry, Proc. of the IEEE MTT-S International Microwave Symposium Digest, Atlanta, GA, 15-20 June 2008, pp. 375-378.

DOI: https://doi.org/10.1109/MWSYM.2008.4633181

[7] T. Sokoll, A. F. Jacob, Self-calibration circuits and routines for low-cost measuring systems, Microw. Opt. Technol. Lett. 50 (2008) pp. 287-293.

DOI: https://doi.org/10.1002/mop.23083

[8] A. Alimenti, N. Pompeo, K. Torokhtii, T. Spina, R. Flükiger, L. Muzzi, E. Silva, Surface impedance measurements on $\mathrm{Nb}_{3} \mathrm{Sn}$ in high magnetic fields, IEEE Trans. Appl. Supercond. 29 (2019) Art. ID 3500104.

DOI: https://doi.org/10.1109/TASC.2019.2892584

[9] A. Alimenti, K. Torokhtii, E. Silva, N. Pompeo, Challenging microwave resonant measurement techniques for conducting material characterization, Measurement Science and Technology 30 (2019) Art. ID 065601.

DOI: https://doi.org/10.1088/1361-6501/ab0e65
[10] M. Scheffler, M. M. Felger, M. Thiemann, D. Hafner, K. Schlegel, M. Dressel, K. S. Ilin, M. Siegel, S. Seiro, Chr. Geibel, F. Steglich, Broadband Corbino spectroscopy and stripline resonators to study the microwave properties of superconductors, Acta IMEKO 4 (2015) pp. 47-52. Online [Accessed 25 September 2020] DOI: https://doi.org/10.21014/acta imeko.v4i3.247

[11] K. Torokhtii, A. Alimenti, N. Pompeo, F. Leccese, F. Orsini, A. Scorza, S. A. Sciuto, E. Silva, Q-factor of microwave resonators: calibrated vs. uncalibrated measurements, J. Phys. Conf. Ser. 1065 (2018) Art. ID. 052027. Online [Accessed 25 September 2020] DOI: https://doi.org/10.1088/1742-6596/1065/5/052027

[12] K. Torokhtii, A. Alimenti, N. Pompeo, E. Silva, Uncertainty in uncalibrated microwave resonant measurements, Proc. of the XXIII IMEKO TC4 International Symposium Electrical \& Electronic Measurements Promote Industry Xi'an, China, 17-20 September 2019, pp. 98-102. ISBN: 978-606-13-5238-8. Online [Accessed 25 September 2020]

URL: https://www.imeko.org/publications/tc4-2019/IMEKOTC4-2019-022.pdf

[13] Embedding/De-embedding, Anritsu Application Note, 2002.

[14] N. Pompeo, K. Torokhtii, E. Silva, Dielectric resonators for the measurements of the surface impedance of superconducting films, Meas. Sci. Rev. 14 (2014) pp. 164-170.

DOI: https://doi.org/10.2478/msr-2014-0022

[15] R. E. Collin, Foundations for Microwave Engineering, WileyIEEE Press, 2001, ISBN: 978-0-780-36031-0.

[16] N. Pompeo, K. Torokhtii, F. Leccese, A. Scorza, S. Sciuto, E. Silva, Fitting strategy of resonance curves from microwave resonators with non-idealities, Proc. of I2MTC 2017 - 2017 IEEE International Instrumentation and Measurement Technology Conf., J. Phys. Conf. Ser., Torino, Italy, 22-25 May 2017, pp. 1-6. DOI: https://doi.org/10.1109/I2MTC.2017.7969903

[17] J. W. Yoon, R. Magnusson, Fano resonance formula for lossy two-port systems, Opt. Express 21 (2013) pp. 17751-17759. DOI: https://doi.org/10.1364/OE.21.017751

[18] P. J. Petersan, S. M. Anlage, Measurement of resonant frequency and quality factor of microwave resonators: Comparison of methods, J. Appl. Phys. 84 (1998) pp. 3392-3402. DOI: https://doi.org/10.1063/1.368498

[19] K. Leong, J. Mazierska, Precise measurements of the Q factor of dielectric resonators in the transmission mode-accounting for noise, crosstalk, delay of uncalibrated lines, coupling loss, and coupling reactance, IEEE Trans. Microw. Theory Tech. 50 (2002) pp. 2115-2127.

DOI: https://doi.org/10.1109/TMT'T.2002.802324

[20] M. Žic, An alternative approach to solve complex nonlinear leastsquares problems, J. Electroanal. Chem. 760 (2016) pp. 85-96. DOI: https://doi.org/10.1016/i.jelechem.2015.11.015

[21] J. J. More, B. S. Garbow, K. E. Hillstrom, User Guide for MINPACK-1, Argonne National Laboratory, Argonne, Ill., 1980.

[22] URL: https://www.anritsu.com

[23] M. Wollensack, J. Hoffmann, J. Ruefenacht, M. Zeier, VNA Tools II: S-parameter uncertainty calculation, Proc. of the $79^{\text {th }}$ ARFTG Microwave Measurement Conference, Montreal, Canada, 22 June 2012, pp. 1-5.

DOI: https://doi.org/10.1109/ARFTG79.2012.6291183

[24] P. Virtanen, R. Gommers, T. E. Oliphant, M. Haberland, T. Reddy, D. Cournapeau, E. Burovski, P. Peterson, W. Weckesser, J. Bright, S. J. van der Walt, M. Brett, J. Wilson, K. J. Millman, N. Mayorov, A. R. J. Nelson, E. Jones, R. Kern, E. Larson, C. J. Carey, I. Polat, Y. Feng, E. W. Moore, J. VanderPlas, D. Laxalde, J. Perktold, R. Cimrman, I. Henriksen, E. A. Quintero, C. R. Harris, A. M. Archibald, A. H. Ribeiro, F. Pedregosa, P. van Mulbregt, and SciPy 1.0 Contributors, SciPy 1.0: fundamental algorithms for scientific computing in Python, Nat. Methods, 17, (2020), pp. 261-272 DOI: https://doi.org/10.1038/s41592-019-0686-2 